\title{
EARLY STAGE MODEL BASED SYSTEM DESIGN UNDER UNCERTAINTIES
}

\author{
Oizumi, Kazuya; Ito, Akio; Aoyama, Kazuhiro
}

The University of Tokyo

\begin{abstract}
System design at the early stage of design plays an important role in design process. Model based systems engineering is seen as a prominent approach for this challenge. System design can be explored by means of system simulation. However, as the system is a complex system, system model tends to have high level of abstraction. Therefore, the models cannot depict every details of the system, which makes optimization unreasonable.

Furthermore, at the early stage of design, there are many uncertainties such as success of technological developments. By properly incorporating uncertain factors in system design, the system can be tolerant. Currently system design is conducted by experienced experts. However, for more complex system, it would be difficult to continue the current practice. Therefore, a method to support design team to make decision in system design is needed.

This paper proposes a computational support for the system design. Design constraints, which seems the core information that design team wants at system design, are modeled. By visualizing constraints quantitatively and intuitively, the proposed method can support design team to conduct system design and design study.
\end{abstract}

Keywords: Systems Engineering (SE), Early design phases, Uncertainty, Simulation, Implicit knowledge

Contact:

Oizumi, Kazuya

The University of Tokyo

Department of Systems Innovation

Japan

oizumi@m.sys.t.u-tokyo.ac.jp

Cite this article: Oizumi, K., Ito, A., Aoyama, K. (2019) 'Early Stage Model Based System Design under Uncertainties', in Proceedings of the 22nd International Conference on Engineering Design (ICED19), Delft, The Netherlands, 5-8 August 2019. DOI:10.1017/dsi.2019.369 


\section{INTRODUCTION}

In design of large scale complex system, system design at the early stage plays an important role as it entails later design process. Thus, system engineering is widely accepted. For mechatronics products, model based systems engineering is seen as a prominent approach for this challenge. Upon constructing system simulation models, system design can be explored and optimised. However, as a complex system includes several domains that are difficult to be modelled, system model usually tends to have high level of abstraction. Therefore, the models cannot depict entire details of the system, which makes optimisation unreasonable.

Furthermore, at the early stage of design, there are many uncertainties such as success of technological developments, contracts with supplier and so on. By properly incorporating uncertain factors in system design, the system can be tolerant.

In practice, system design relies on system design experts who experienced design of the product for a long time. However, this practice would not last long as products would be larger and more complex. Therefore, a method to support system design under uncertainty is needed.

\section{RELATED WORKS}

System design has been discussed in both academics (Ulrich and Eppinger, 2011) and industrial practice (INCOSE 2015, SEBok, 2015). (Parnell et al., 2014) described that trade studies play a central role in decision management and can be applied throughout a systems lifecycle. Further, (Cilli and Parnell, 2014) and (Edwards et al., 2015) proposed the process of trade study in system design stage. In these implementations a common architecture of subsystems is defined for the system of interest, variation of the subsystems within this architecture enables alternative system designs to be generated and assessed through simulations. In (Edwards et al., 2015), performance measures are combined by a value function, which uses weighting between measures that enable Multi Objective Decision Analysis (MODA). It implies that optimum design can be obtained through simulations.

However, especially for early stage of design, construction of such value function is difficult as designers should not know how to weigh performance measures as there are so many uncertainties.

To handle uncertainties, set-based design has been introduced by (Wood and Antonsson, 1989). By giving ranges to design parameters, uncertainties are incorporated. By combining different values of design parameters, set of design solutions are generated. When conditions of performance requirements are specified, sets are narrowed. Uncertainties are dealt as how ranges of design parameters are narrowed.

Computational cost of system simulation model can be too large as it depicts holistic behaviour of the system. To practically conduct optimal system design, computational cost needs to be controlled. For instance, set-based design requires much computation, the model needs to be light. Christophea et al., 2008) proposed to use dimensional analysis to simplify the system models, which is called dimensional analysis conceptual modelling (DACM). DACM enables qualitative investigation into the system at early stage of design, which means uncertainty in early stage is dealt by abstracting the system. Therefore, quantitative optimisation is not incorporated. (Woldemariam et al., 2018) further customized it to quantitative system optimization. However, by incorporating quantitative optimisation, uncertainty is not considered. While DACM is applicable to single objective optimization, product system design is often multi-objective. Furthermore, optimality itself is quite obscure.

Actual system design needs to be conducted under the condition that system simulation model cannot incorporate much information for the reasonable decision making. Uncertainty is not solved but handled so that the effects of uncertainties should not bring about failure of the design. Therefore, a method to handle information not expressed in simulation models and to make the system tolerant to uncertainties needs to be developed.

\section{APPROACH}

This paper proposes a process of system design along with design study by means of system simulations. First of all, types of information involved in system design are discussed. Useful information that experienced designers acquire through design study is mathematically modelled. 
These types of information are presented by a computer to help designers to conduct system design effectively. By incorporating types of information not expressed by simulation model, which are known by designers, system design process is proposed. Therefore, the focuses of this paper are as follows.

- How information acquired through design study could be modelled mathematically;

- How information not expressed by simulation model can be considered in system design.

The left side of Figure 1 depicts current information processing in system design along with design study. Here, it is assumed that system simulation and conditions are available. Thus, designers can conduct design study by changing design parameters. By executing several simulations, designers can acquire information useful to decide values of design parameters or assumptions for further design studies. In the current way, how to change design parameters and interpretation of the simulation results depends on designers' expertise. As a product increases its scale and complexity, it would be difficult for designers to conduct design study properly.
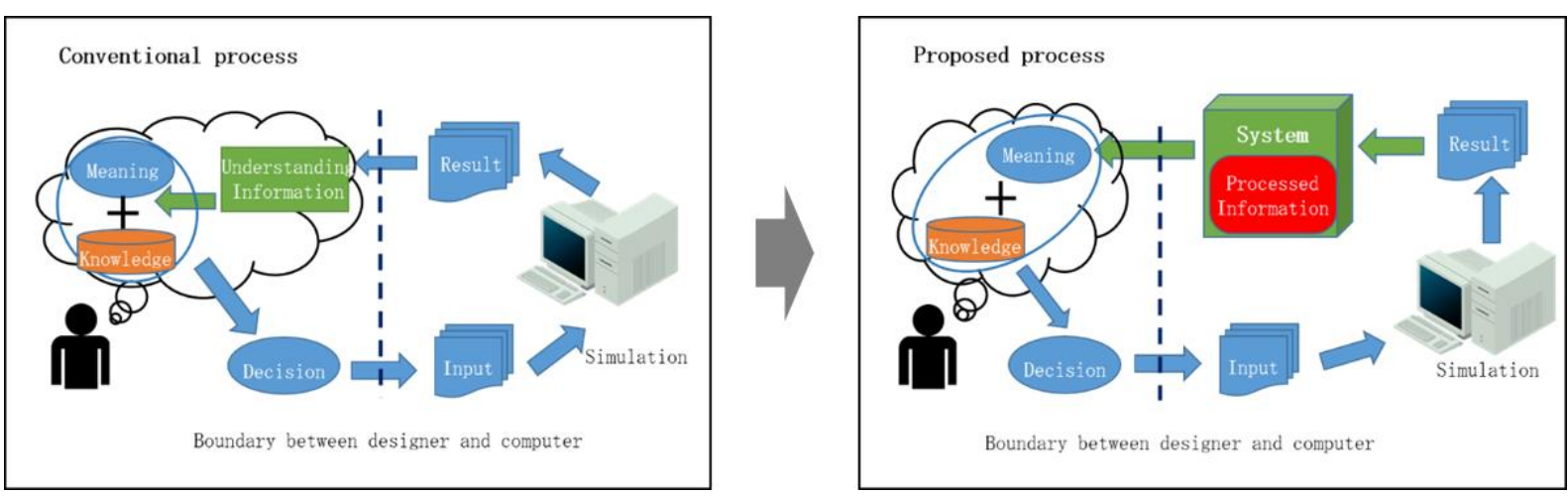

Figure 1. Information processing in system design along with design study

To solve this problem, this paper proposes a process as shown in the right side of Figure 1. In the proposed process, information designers want to acquire through design study is elicited.

The proposed process does not employ mathematical optimisation. Though the process seems optimization by human brain, there are strength for taking this process. As discussed earlier, there are some uncertainties and other information that are not expressed by the system simulation models. The proposed process incorporates such types of information in system design. As shown in the right side of Figure 1, designers make decisions on how design study is done or how system design should be.

While deciding range of design parameters to change, uncertainties of developments can be incorporated. If it is uncertain whether a component is developed as decided in system design, its parameter should have wider range. If required performances are fulfilled by any value of the parameter in the range, the design is readily considering the uncertainty.

Furthermore, types of information not expressed by the system simulation models can be considered by intervention by designers. For example, combination of design parameters' values can reflect sizing constraints. Likewise, preference for choosing value of design parameter can reflect concern for cost. By following the proposed process for system design, rational behind the adopted system design could be elicited and shared within design team. Therefore, the process is not just optimization of system design but also includes an explanation of reasons why the system design is adopted.

\section{DESIGN STUDY AT SYSTEM DESIGN STAGE}

System design is conducted along with design study that makes use of system simulation models. To decide system design, designers execute simulations with different sets of parameters. Through design study, designers acquire information that is useful for deciding system design. In this section, types of information involved in system design along with design study is discussed.

\subsection{Information existing before design study}

In this paper, following types of information are assumed to be available before design study.

1. System simulation model

This paper assumes that component based simulation model of a product is readily available.

Here, "component based" means that physical equations or behaviours are encapsulated to each 
component. Modelica is well-known system simulation modelling language for component based simulation model. Same kind of encapsulation can be implemented in other means such as Matlab/Simulink. Existence of the simulation model assures known physics to be considered at system design, such as relationships between parameters, variables, and performance measures. As system simulation model is abridged expression of a product, there are several phenomena and other factors that are not expressed in models.

2. Conditions

System design is to configure design of each component so that required performances of a product are satisfied. Satisfaction of the performance is evaluated by measuring several performance measures. Thus, required levels of performance measures are given as conditions of system design.

Besides performances, there are several factors working as conditions. For instance, cost, technologies, size, and development strategy could also be conditions. Even required performances are satisfied, if required specifications are not technologically achievable, it would not be realized. Likewise, if it exceeds cost limit, the company cannot proceed the development.

3. Knowledge of designers

While system simulation model is made of knowledge of designers, the model cannot contain all the available knowledge. Knowledge is not only about technology or physics, but also about economy, project management and so on. Design study is an interplay between simulation and knowledge of designers. While knowledge about physics consists of simulation models, there is a rationale behind what is and how it is modelled. To execute a simulation, parameters needs to be set, such as initial conditions or variations of design parameters. There is a rationale behind the choice of these parameters as well. Further, to learn from simulation, the rationale needs to be understood. Therefore, a rationale and simulations are strongly tied together. Without a designers' rationale, simulation is merely a mathematic calculation.

4. Simulation results

By executing multiple simulations, designers can obtain several results. However, without a rationale to interpret those results, what simulations can output are merely time-series values of variables.

\subsection{Information acquired through design study}

As there is an interplay between simulation and a rationale, designers can acquire new information useful for deciding system design. The rationale includes several intentions such as improvement of functionality, productivity and so on. In this paper, as the focus is on how to determine system design that is tolerant to uncertainties at the early stage of design, the concern is what designers intend to make the system tolerant.

Here, types of information that should be acquired for the determination of system design are discussed. The information needs to explain why the determined system design seems relevant for making product tolerant to uncertainties. There are several different types of uncertainties in product development; uncertain results of on-going developments, uncertain future changes on requirements, and uncertain phenomena that may occur in a product. Tolerant system design to uncertainties can be understood as a set of allocated specifications of components that can satisfy requirements with no or minimum changes to the original design when uncertain factors are revealed.

In three types of uncertainties, this paper focuses on uncertain results of on-going developments. As the system design is conducted at early stage when development of technologies installed on the product is still going on, levels of technologies available at the release of the product have some extent of uncertainties. Ideal system design should satisfy requirements regardless ranging resultant specifications of components that are under development.

As a product comprises several components, while some components have uncertainties due to developments, others should have rather certain specifications. Thus, the concern would be what range can be allowed for each uncertain resultant specification of a component. By determining specifications of both certain and uncertain components properly, larger allowance can be allocated to uncertain components, which makes the product tolerant to uncertainties.

To explore possible allocation of design ranges to each specifications of components design study is conducted. What designers concern about is how determination of certain specifications affects system performances and constrains ranges of other specifications. Following two types of information can be acquired through design study by means of system simulation. 
1. Design constraints

As a performance measure of a product is achieved by harmonization of several component design, when a specification of a component is determined, other specifications of components may be affected. These effects could be either positive or negative. For example, by choosing efficient engine, efficiency requirements for other powertrain could be released. In contrast, as a larger engine is chosen, smaller radiator cannot be chosen as large heat radiation capacity is required. In short, design of a certain part of a product constrains design of other part. Design study is conducted to evaluate these constraints. While dealing specifications of components as design parameters, different values of parameters are tested to see where these constraints exist. To make a product tolerant to uncertain components, constraints to uncertain components should be loosen.

2. Sensitivities

Another concern is how changing value of design parameter affects performances measures, which is usually called a sensitivity analysis. As sensitivities of performance measures may be affected by other design parameters as well, changes of sensitivities should be observed by changing other design parameters. To make a product tolerant to uncertain components, sensitivities of performance measures to specifications of the component should be minimized. To detail the analyses, not only performance measures but also variables that depict product system's behaviour should also be observed.

\section{MODELING OF INFORMATION ACQUIRED THROUGH DESIGN STUDY}

To realize the process proposed above, information that designers want to acquire through design study is mathematically modelled. As discussed earlier, design constraints and sensitivities seems dominant information to decide system design. Thus, these two types of information are computationally elicited. To elicit design constraints and sensitivities, design of experiments is employed. As design of experiments takes several different levels for each design parameter and shows how evaluation measure responds to design parameters, which is called sensitivities, it is suitable for design study. Figure 2 shows elicitation of sensitivities.
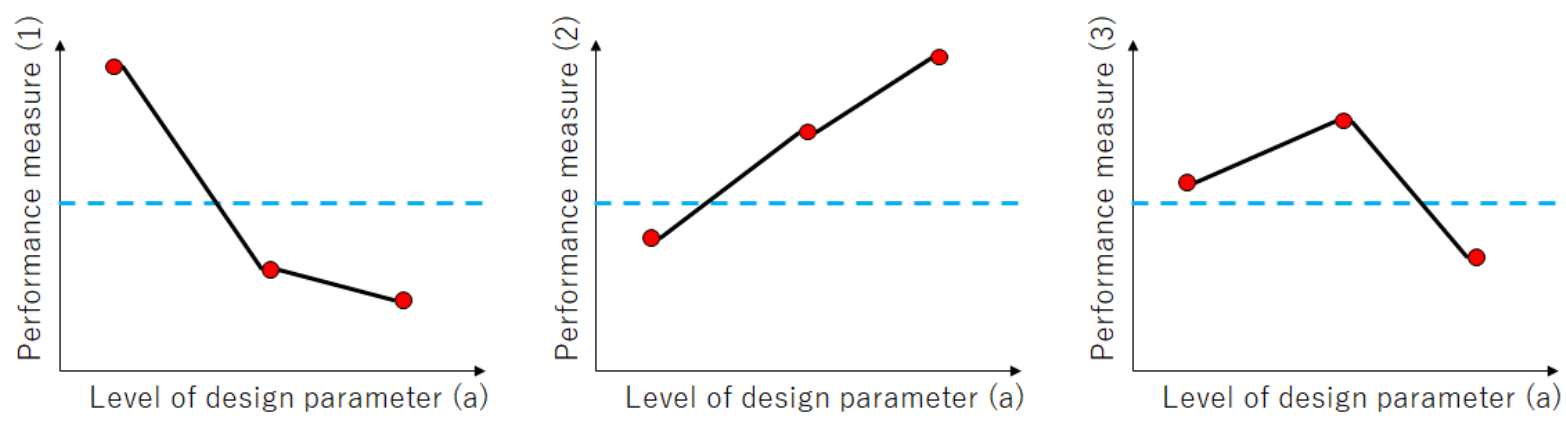

Figure 2. Sensitivities acquired by design of experiments

\subsection{Quantification of design constraints}

To elicit design constraints, it is quantified from simulation results whose cases are created by design of experiments. As explained above, when a value of a design parameter is determined, it may constrain possible values of other design parameters. By choosing different value for the design parameter, these constraints may change. Thus, determination of those design parameters who pose strong constraints should be deliberated so that a design parameter that has an uncertainty would not be strongly constrained. To help designers to specify such constraining design parameters, computer should suggest existence of possible strong constraints. Therefore, strength of constraints should be quantified.

To evaluate how each design parameter affects a product system, several sets of different levels of design parameters are tested. To reduce the number of simulations so that computation cost would be reasonable, design of experiments is employed. As a result, number of test cases and their simulation results by whom performance measures are evaluated can be obtained as shown in Figure 3. Figure 3 shows the plots of test cases. The horizontal axis denotes levels of a design parameter (a) while the vertical axis denotes values of a performance measure (1). As each plot contains levels of other design parameters respectively, by choosing a certain level for a specific design parameter (b), number of 
cases that satisfy required level of the performance measure may vary. As shown in Figure 3, depending on a choice of level of the design parameter (b), level of the design parameter (a) that has higher potential to satisfy required level of the performance measure (1) would be different. When the design parameter (b) takes level $\langle 1\rangle$, the performance measure (1) is more likely to be satisfied if the design parameter (a) takes level $\langle 3\rangle$ and not likely to be satisfied by level $\langle 1\rangle$. In this case, the design parameter (a) is constrained by design parameter (b). Here, extents of constraint are different among levels of a design parameter. In the case of Figure 3, when the design parameter (b) takes level $\langle 2\rangle$, the design parameter (a) is less constrained, as likelihoods of satisfying the performance measure (1) are almost same among levels of the design parameter (a).
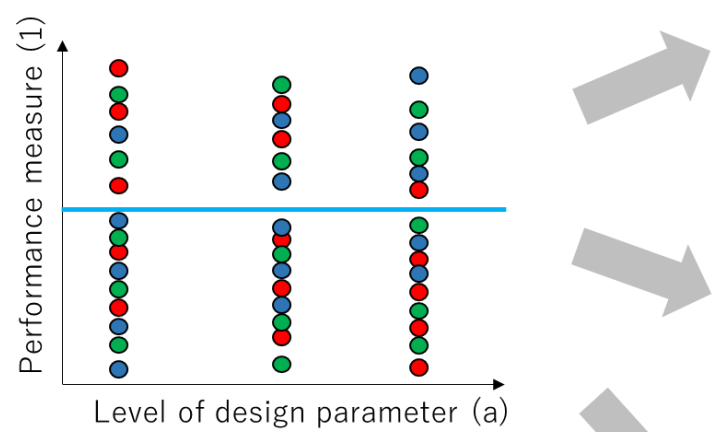

Level of design parameter (a)

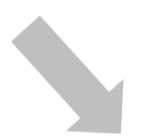

Result when design parameter $b$ is

$\circ \begin{array}{lllll}\circ & 1 & 2 & \circ & 3\end{array}$
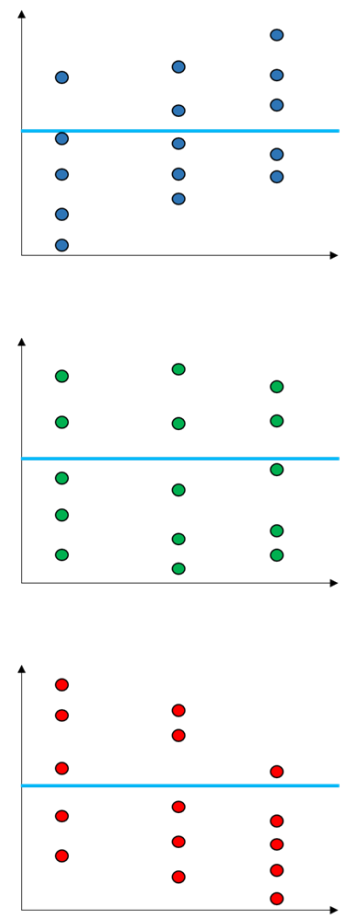

Figure 3. How design parameter (a) is constrained by design parameter (b)

To express extent of design constraint, constraint degree is defined and calculated by the following equation (1).

$$
C_{i j k l}=\sum_{m}\left(n_{i j k l m}{ }^{*}-n_{i j k l m}\right) /(M-1) \sum_{m} n_{i j k l m}
$$

Basically, equation (1) calculates how jth design parameter is constrained by $k$ th design parameter. Here, $C$ denotes constraint degree of $j$ th design parameter when $k$ th design parameter takes $l$ th level, in view of $i$ th performance measure. $n$ denotes number of test cases that satisfy required level of $i$ th performance measure when $k$ th design parameter takes $l$ th level and $j$ th design parameter takes $m$ th level. $m^{*}$ denotes level of $j$ th design parameter that has largest number of satisfactory test cases among levels of $j$ th design parameter. $M$ denotes number of levels $j$ th design parameter can take.

If number of test cases that satisfies $i$ th performance measure would not change at all regardless the level of $j$ th design parameter, constraint degree is 0 , which means $j$ th design parameter is not constrained when $k$ th design parameter takes $l$ th level. On the other hand, if $i$ th performance measure is satisfied only when $j$ th design parameter takes $m$ th level, constraint degree is 1 , which means $j$ th design parameter is completely constrained when $k$ th design parameter takes $l$ th level. The extents of constraints are evaluated between 0 and 1 .

\subsection{Visualization of design constraints and sensitivities}

The former section explained the calculation method of design constraints and sensitivities. However, as a product increases its scale and complexity, it would be difficult for designer to find a right place to start consider about. Therefore, a method to visualize significant constraints and sensitivities is necessary so that designers can process system design and design study properly. This paper proposes ways to visualize design constraints and sensitivities respectively. 


\subsubsection{Network description of design constraints}

Even though the constraints degrees are calculated by equation 1, they depict a design parameter is constrained by another design parameter in a performance measure, which is microscopic evaluation of a part of a system. The concern of designers is which design parameters should be the first to study, which is rather macroscopic than the constraint degrees. Therefore, macroscopic constraint should be visualized. As design constraints exist between design parameters, network description can be utilized as shown in Figure 4. Figure 4 shows networks of design parameters whose links denote constraints. However, there are several constraints between design parameters, as constraint degree is calculated for each performance measure and each level of constraining design parameter. Thus, for the network description, information of design constraints needs to be condensed. If a design parameter is strongly constrained by another design parameter through even one performance measure, the constraints between those design parameters are strong. Therefore, taking maximum constraint degree as representative strength of constraints seems relevant. Thus, the weight of links between design parameters are given by maximum constraint degree.

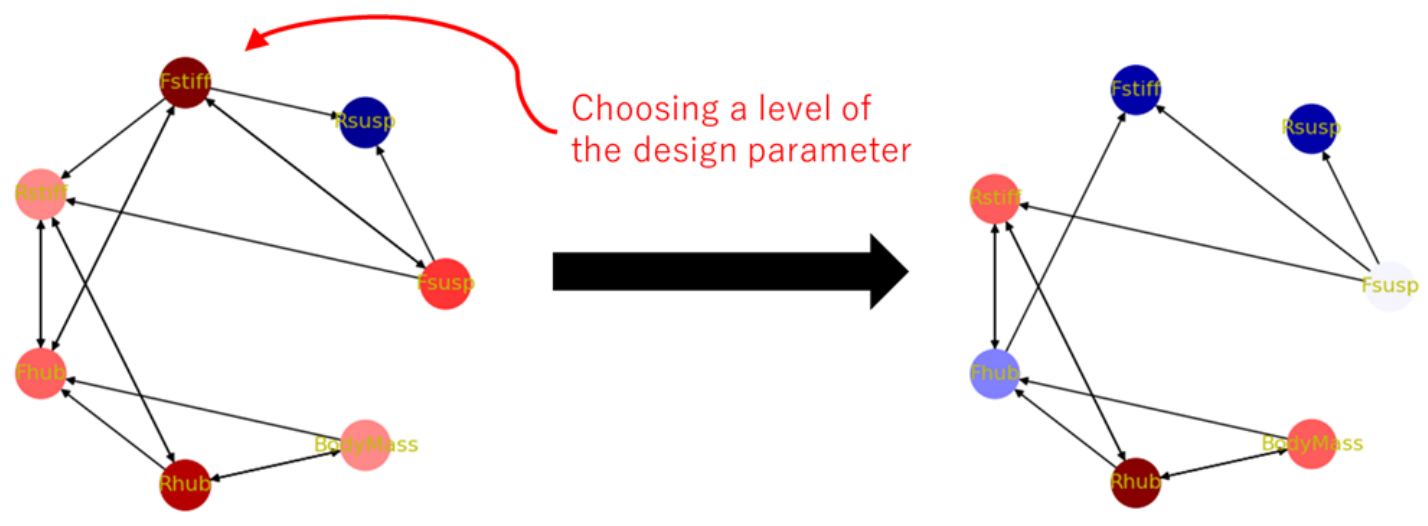

Figure 4. Constraint network of design parameters that changes by choosing a level

As a product is integral where most design parameters are related to each other, it is likely that almost all design parameters constrains others at any rate. If all constraints are visualized in the network, the network would be too dense that significant constraints cannot be specified. Therefore, constraints to be visualized should be limited. By applying threshold to the constraint degrees, number of visible links can be reduced.

To stabilize the system, design parameters that pose strong constraints should be levelled properly. Therefore, significant design parameter should be informed as well. If the network is constructed as explained above, network analyses can be applied. In this case, page rank algorism is applied to specify significant design parameters. To visualize significance, colours are given to nodes (red: significant).

\subsubsection{Chart description of sensitivities}

As discussed above, another type of information that designers want from design study is sensitivities. In design of experiment method, sensitivities are usually visualised as a set of charts. This expression gives both intuitive and quantitative information to decide significance. Thus, this research inherits the chart description of sensitivities in design of experiment.

\subsubsection{Dynamic reconstruction of constraint network and sensitivity charts}

By choosing right level for a design parameter, constraints are mitigated. Therefore, depending on the choice of a design parameter's level, constraint network and sensitivity charts should also be changed to show designers to see how each choice affects the system. By dynamically reconstructing constraint network and sensitivity charts accordingly to levels chosen for design parameters, comparison between different choices of levels is intuitively supported.

As explained in former section, constraint degree is calculated for each level of design parameter. Therefore, for the design parameters whose levels are chosen, by visualizing only constraint degree with chosen level, it is possible to reconstruct constraint network (Figure 4). Likewise, as for sensitivity charts, as it depicts averages of test cases, by counting only the cases with selected level of the design parameters, sensitivity charts can be reconstructed as well. 


\section{PROCESS OF SYSTEM DESIGN ALONG WITH DESIGN STUDY}

The flow of the proposed method is shown in Figure 5. First, required levels of performance measures are set. Second, levels of design parameters are set. Then the simulations are executed. From the simulation results, constraint degrees are calculated. And, constraint network and sensitivity charts are constructed. As the information is provided to designers, they investigate into results and explore possible design solutions by means of interactive visualization of constraint network and sensitivity charts. As a result, levels of design parameters are renewed. It can be both partially narrowed system design and further design studies. In case, certain level of a design parameter seems relevant and can be specified in smaller range, levels can be narrowed for the next simulations. In contrast, if levels of a design parameter seem irrelevant, different range should be tested to see which range is more relevant. If it is allowed, required levels of performance measures can be renewed as well. While repeating those processes, a system design is narrowed enough to be adopted as a system design.

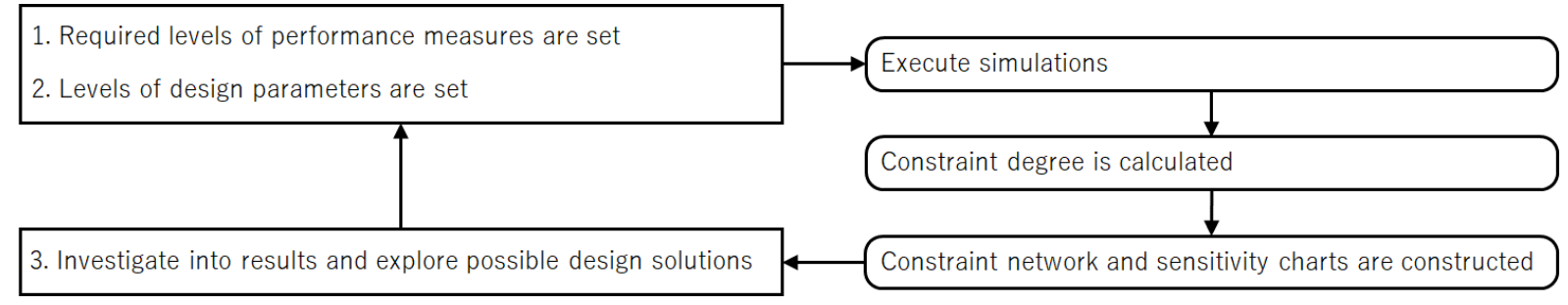

Figure 5. Flow of the proposed method

\section{APPLICATION}

The proposed method is applied to the design of an automobile suspension system. As shown in Figure 6, The automobile has 7 design parameters. Performance measures are 4 metrics regarding shock absorption, vertical/horizontal shake amplitudes and cool times of vertical/horizontal shakes.

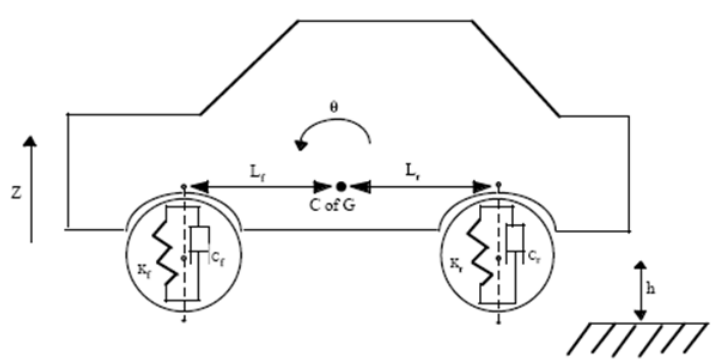

- Design parameters

Fhub,Rhub : Distance of front/rear hub from center of gravity[m] BodyMass : Mass of the automobile [kg]

Fstiff,Rstiff : Stiffness of front/rear suspension $[\mathrm{N} / \mathrm{m}]$

Fsusp,Rsusp : Damping rate of front/rear suspension [N sec/m]

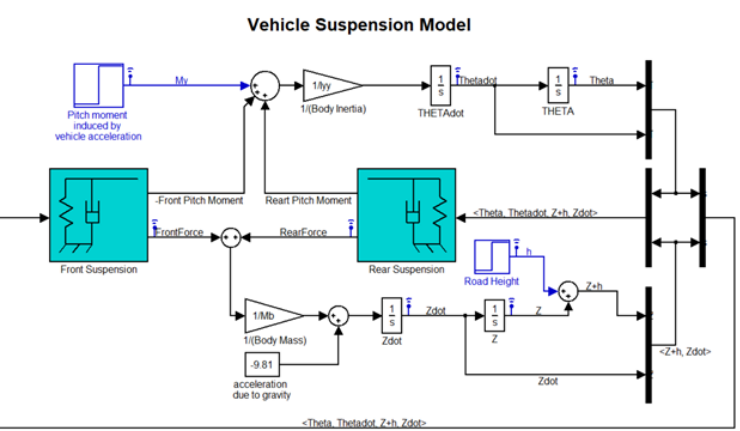

- Performance measure

ShakUD : Amplitude of vertical shake $(\mathrm{dZ} / \mathrm{dt})<0.1$ ShakFR: Amplitude of horizontal shake $(\mathrm{d} \theta / \mathrm{dt})<0.01$ CTimeUD : Cool time of vertical shake $[\mathrm{sec}]<0.12$ CTimeFR : Cool time of horizontal shake [sec] $<0.12$

Figure 6. Applied automobile and its simulation model

Table 1. Initial settings of the design parameter levels

\begin{tabular}{|l|c|c|c|l|c|c|c|}
\hline $\begin{array}{l}\text { Design } \\
\text { parameter }\end{array}$ & Level 1 & Level 2 & Level 3 & $\begin{array}{l}\text { Design } \\
\text { parameter }\end{array}$ & Level 1 & Level 2 & Level 3 \\
\hline $\begin{array}{l}\text { Fsusp } \\
{[\mathrm{N} /(\mathrm{m} / \mathrm{s})]}\end{array}$ & 1900 & 2300 & 2700 & $\begin{array}{l}\text { Fhub } \\
{[\mathrm{m}]}\end{array}$ & 0.8 & 1 & 1.2 \\
\hline $\begin{array}{l}\text { Rsusp } \\
{[\mathrm{N} /(\mathrm{m} / \mathrm{s})]}\end{array}$ & 1800 & 2200 & 2600 & $\begin{array}{l}\text { Rhub } \\
{[\mathrm{m}]}\end{array}$ & 0.8 & 1 & 1.2 \\
\hline $\begin{array}{l}\text { Fstiff } \\
{[\mathrm{N} / \mathrm{m}]}\end{array}$ & 20000 & 25000 & 30000 & $\begin{array}{l}\text { BodyMass } \\
{[\mathrm{kg}]}\end{array}$ & 1100 & 1300 & 1500 \\
\hline $\begin{array}{l}\text { Rstiff } \\
{[\mathrm{N} / \mathrm{m}]}\end{array}$ & 20000 & 25000 & 30000 & & & & \\
\hline
\end{tabular}


Table 1 shows the initial setting of levels of design parameters. Then the simulations are executed for test cases generated by design of experiments. By applying the proposed method to results of simulations, constraint and sensitivity information is constructed. Figure 7 is the several constraint network shown in the first round simulations. In this case study, the body mass has large uncertainty because it is revealed when masses of constituting parts are specified. Therefore, by allowing $60 \mathrm{~kg}$ range to the body mass, this uncertainty can be handled. That is, if performance measures are fulfilled even the body mass varies in $60 \mathrm{~kg}$ range, the system design is tolerant to this uncertainty.

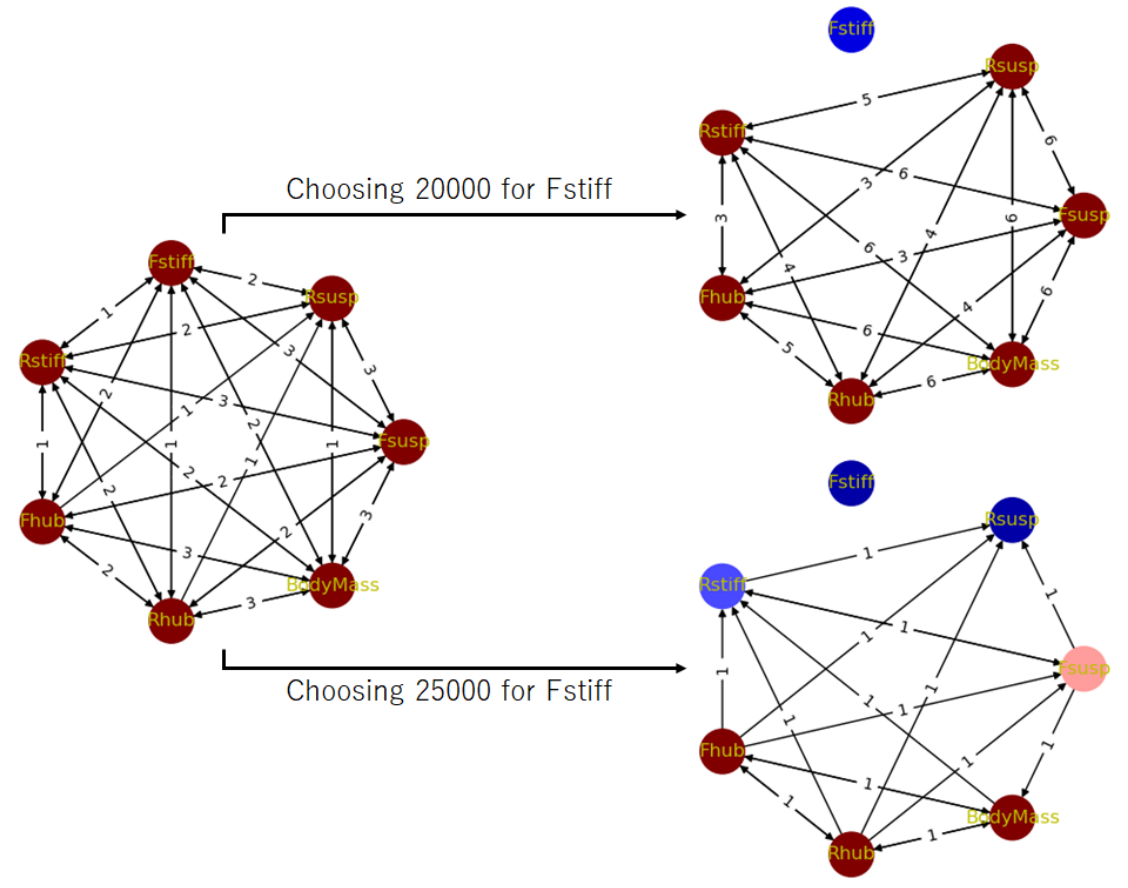

Figure 7. Changing constraint network by specifying level of a design parameter (first round)

The constraint network shown at the left side of Figure 7 is the first constraint network, which all levels of design parameters are taken into consideration in calculating constraint degrees. By specifying a level for each design parameter, the constraint network is changed.

One of the most significant change of the network was observed when changing the stiffness of the front suspension. The two constraint networks shown at the right side of Figure 7 are shown when the stiffness of the front suspension is specified as 20000 and 25000 respectively. As a result, the stiffness of the front suspension is decided to be around 25000 at this point. By observing sensitivity charts, the range of the parameter for the second round simulations is decided to be from 25000 to 30000 .

At this point, the constraint network is shown as the right bottom one in Figure 7. It suggests three design parameters (brown) are still significantly constraining other design parameters. Because distance of rear hub from centre of gravity also constrains other design parameters when it is short, distance was decided to be between 1.15 and 1.19.

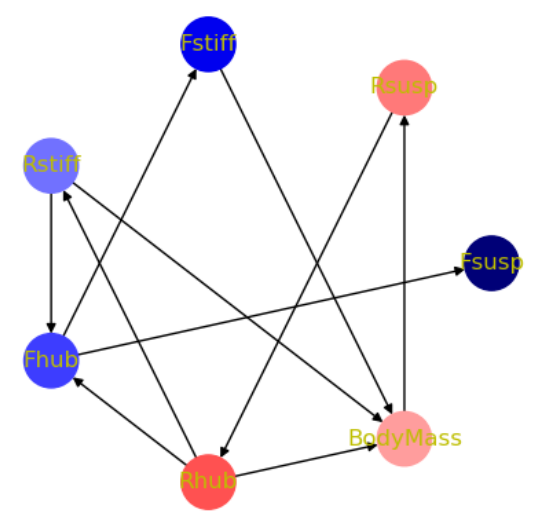

Figure 8. Constraint network constructed at the second round 
Likewise, ranges of other design parameters are decided. By adopting the decided ranges of the design parameters at this round, the second round simulations were executed. As a result, constraint network is constructed as shown in Figure 8.

By comparing constraints networks of the first and the second rounds, it was shown that constraints were relieved. As distance of rear hub from centre of gravity still constrains other design parameters, it was decided to be further narrowed to between 1.16 and 1.18. In this round, dumper rate of rear suspension was also suggested to be significant. By taking cost into account, levels of the dumper rate are narrowed to between 1960 and 2040. Likewise, the body mass, which is suggested as significant, was also narrowed to between 1170 and 1230 . As explained above, body mass is uncertain and needs to be allowed to have $60 \mathrm{~kg}$ range. Therefore, this is smallest possible range. If all required performance measures are fulfilled while keeping this range, the system design can be tolerant to uncertain body mass.

By adopting decided ranges of the design parameters at this round, the third round simulations were executed. As demonstrated above, through repeating the rounds, proposed method showed information useful for designers to narrow down a system design solution while taking information not described in the simulation model such as cost and technical uncertainties.

\section{DISCUSSION AND CONCLUSION}

Through the application demonstrated in the former section, constraint degrees appeared to be useful for deciding range of design parameters. As design constraints on this level of abstraction is mostly about compensating performance measures worsen by choice of other design parameters, sensitivity perspective is mostly taken into account. Therefore, how to incorporate constraint perspective into system design appeared to be a key for successful system design.

Furthermore, mechanism to taking information not described in simulation models are also important to obtain reasonable system design. There are three reasons for this. First, optimisation of system design may result in infeasible design as the information that can be describe in system simulation models are quite limited. Second, by showing results in each round, designers can acquire information that can foster knowledge. Third, uncertainty can be expressed by how designers narrow down range of design parameters. The proposed method realized them by taking intervention by designers in each round.

In conclusion, the proposed method was appeared to be useful for system design under uncertainties. By taking several round to narrow down ranges of design parameters, it was possible to allow room for taking uncertainty into account. Furthermore, constraint degrees can be a good indicator to decide how to narrow down these ranges. As the proposed method is applied only to an imaginary case to demonstrate its usefulness, further validation with actual industrial cases are needed. Currently, it is applied to a design of an automobile in cooperation with an industrial partner.

\section{REFERENCES}

Christophea, F., Sellb, R. and Coatanéaa, E. (2008), “Conceptual design framework supported by dimensional analysis and System Modelling Language”, Estonian Journal of Engineering, Vol. 14 No. 4, pp. $303-316$.

Cilli, M.V. and Parnell, G.S. (2014), "Systems engineering tradeoff study process framework", 24th INCOSE International Symposium, Las Vegas, NV.

Edwards, S., Cilli, M.V., Peterson, T., Zabat, M., Lawton, C. and Shelton, L. (2015), "Whole Systems Trade Analysis", 25th INCOSE International Symposium, Seattle, WA.

INCOSE (2015), Wiley: INCOSE Systems Engineering Handbook: A Guide for System Life Cycle Processes and Activities, 4th Edition, Wiley.

Parnell, G.S., Cilli, M.V. and Buede, D. (2014), "Tradeoff Study Cascading Mistakes of Omission and Commission", 24th INCOSE International Symposium, Las Vegas, NV.

SEBoK. (2015), Guide to the Systems Engineering Body of Knowledge (SEBoK). [online]. Available at: https://www.sebokwiki.org/wiki/Guide_to_the_Systems_Engineering_Body_of_Knowledge_(SEBoK) (accesses 1/Dec/2018)

Ulrich, K. and Eppinger, S. (2011), Product Design and Development (5th edition), McGraw-Hill, Irwin, New York.

Woldemariam, E.T., Coatanéa, E., Wang, G.G., Lemu, H.G. and Wu, D. (2018), “Customized dimensional analysis conceptual modelling framework for design optimization-a case study on the cross-flow micro turbine model", Engineering Optimization, https://doi.org/10.1080/0305215X.2018.1519556.

Wood, K.L. and Antonsson, E.K. (1989), "Computations with Imprecise Parameters in Engineering Design", Transactions of the ASME, Journal of Mechanisms, Transmissions and Automation in Design, Vol. 111 No. 4, pp. 616-625. 